\title{
Assessment Framework of Provincial Carbon Emission Peak Prediction in China: An Empirical Analysis of Hebei Province
}

\author{
Wei Li*, Lei Du \\ School of Economics and Management, North China Electric Power University, Baoding, Hebei, China
}

Received: 19 July 2018

Accepted: 30 October 2018

\begin{abstract}
Since China claimed to achieve carbon emission peak around 2030 in the "China-U.S. Joint Presidential Statement on Climate Change," whether or not the target can be accomplished has become the focus of discussion. Thus, the aim of this study is to forecast the carbon emissions peak of Hebei Province in China (as a case study) for the period of 2016-2030 through the historical data of 1990-2015 using the STIRPAT model and GA-BP (BP neural network based on genetic algorithm) model. We choose the proportion of coal consumption, population, urbanization rate, energy intensity, per capita GDP (replaced by GDP in the GA-BP model) and the proportion of services as the independent variables, and set 9 scenarios in the light of different increment speeds of these variables during 2016-2030. Results show that the ranges of estimated carbon emission peaks are 784.1635-1,007.2901 million tons in the STIRPAT model and 702.7465- 702.8144 million tons in the GA-BP model, with corresponding peak years all in or before 2030. Moreover, a comparative study of the STIRPAT and GA-BP models reveals that the GA-BP model estimates carbon emissions more accurately than STIRPAT; however, the STIRPAT model is more precise on the prediction of carbon emission peak years.
\end{abstract}

Keywords: STIRPAT, genetic algorithm, BP neural network, carbon emission peak, China

\section{Introduction}

Greenhouse gas emission reduction has become the focus of today's research. In December 2009 the Chinese government proposed targets aimed at controlling greenhouse gas emissions. More specifically, it was decided that $\mathrm{CO}_{2}$ emissions per unit gross domestic product (GDP) in China should be cut by $40-45 \%$ in 2020 compared to 2005. Then China and the United

*e-mail: author: ncepulw@126.com
States jointly issued the "China-U.S. Joint Presidential Statement on Climate Change" in November 2014 [1], and China claimed to peak $\mathrm{CO}_{2}$ emissions around 2030 and would endeavor to reach the carbon emission peak as soon as possible. Besides, China also made climate change action included in the " $13^{\text {th }}$ Five-Year" Development Planning [2]. Since then, whether or not the target can be accomplished has become the focus of discussion.

Hebei, as a large economic and energy consumption province, is located in the Bohai Sea Economic Zone and the Beijing Tianjin Hebei Economic Circle, with rich fossil energy and oil field resources [3]. Since the 
founding of new China, especially since the reform and opening up, Hebei has formed a multi-pillar industrial structure dominated by steel, coal, the chemical industry, and equipment manufacturing, as well as other resource-intensive and heavily polluting industries. In past years, with the economic growth of Hebei, the waste of energy has become more and more serious, which has caused many ecological environmental problems, such as the haze and smog that has caused great harm to society and individual health. Moreover, possessing a lot of high energy-consuming industrial enterprises, Hebei is facing many issues such as the upgrading industrial structure, enterprise transformation, etc. Therefore, the " $12^{\text {th }}$ Five-Year" Greenhouse Gas Emission Control Program in Hebei (2012.09) has proposed that, compared with 2010, Hebei's carbon emissions per unit of GDP should be reduced by $18 \%$ until 2015, and the energy consumption per 10,000 yuan should be reduced to 1.3 tons of standard coal. Indubitably, the control of carbon emissions in Hebei is imperative.

Much researchhas focused on $\mathrm{CO}_{2}$ emissions. Wang $\mathrm{P}$ et al. analyzed the impacts of an emissions trading scheme policy on carbon emissions in Guangdong Province using a dynamic CGE model [4]. Lu et al. and Zhang et al. investigated the carbon emissions of industries in China using the complete decomposition technique and Malmquist-type index, respectively [5, 6]. Monoj et al. reviewed and studied the current methods in the field of $\mathrm{CO}_{2}$ capture and separation [7]. Lajunen et al. evaluated $\mathrm{CO}_{2}$ emissions of different types of city buses [8].

As for the analysis of influencing factors of carbon emissions, $\mathrm{Xu}$ et al. found that energy efficiency can significantly impact $\mathrm{CO}_{2}$ emissions, followed by private vehicles and urbanization [9]. Tang et al. found that in Vietnam, energy consumption and income would promote $\mathrm{CO}_{2}$ emissions, but the square of income had the opposite impact [10]. Besides, Özbuğday et al. found that the scale of economic activity and industrialization would lead to a significant increase in $\mathrm{CO}_{2}$ emissions [11]. Karmellos et al. used an LMDI-I method to estimate five driving factors of $\mathrm{CO}_{2}$ emissions (level of activity, electricity intensity, electricity trade, efficiency of electricity generation and fuel mix) from the power sector, and results showed that electricity intensity had the domain effect [12].

The IPAT and STIRPAT (stochastic impacts by regression on population, affluence, and technology) models have been mainly used in the study of $\mathrm{CO}_{2}$ emissions [13]. Specially, Zhaohua Wang et al. used an improved STIRPAT model to analyze the influences of urbanization level, economic level, industry proportion, tertiary industry proportion, energy intensity and R\&D output on $\mathrm{CO}_{2}$ emissions in Beijing, China [14]. Put slightly differently, the STIRPAT model used by Ping Wang et al. also examined the factors of population, energy consuption structure and foreign trade degree [15]. Yanan Wang et al. employed STIRPAT to examine the impact of population, per capita consumption, energy intensity, urbanization and aging population on $\mathrm{CO}_{2}$ emissions by adopting panel data of 30 provinces from 1997 to 2012 [16]. Klaus Hubacek et al. assessed population, lifestyle, technology and their associated $\mathrm{CO}_{2}$ emissions in China [17]. Chuyu Xia et al. made a scenario analyses of low-carbon transformation for Zhejiang province in China [18]. Besides, the STIRPAT model has also been applied in other fields. Chunfu Zhao et al. explored the influencing factors of population, affluence, urbanization level, and diet structure on agriculture products-related water footprint change [19]. Muhammad Shahbaz et al. studied the relationship between urbanization and energy consumption in the case of Pakistan [20], while Lei et al. used the STIRPAT model to analyze five types of decomposed energy consumption [21].

Artificial neural network (ANN) has been widely applied as an intelligent model. Based on ANN, some papers have made predictions for wind power [22], wind speed [23], district-level electricity demand [24] and the water-alternating- $\mathrm{CO}_{2}$ process [25]. Alireza Taheri-Rad et al. simulated the energy flows for the production of various paddy rice cultivars [26]; Nadya et al. simulated the relationship between spectral profiles and hardness values [27]; Raul et al. modelled the electric arc furnace [28]. Besides, Marjan et al. applied ANN in the field of chemistry [29], and Morse et al. in the field of composite panels [30]. Based on the application of artificial neural network optimized by genetic algorithm, Hamzeh Soltanali et al. simulated the energy flows of kiwifruit production [31]; Rashidi et al. made a thermodynamic analysis of the ejector refrigeration cycle [32]; and Hamid et al. forecasted the HAZ and temperature distribution of the specimens [33].

In this paper, the combination use of STIRPAT model and the BP neural network model based on genetic algorithm (GA-BP) are applied to predict the $\mathrm{CO}_{2}$ emissions peak of Hebei province, and the theories and methods used in this paper are of referential significance for China and other provinces.

\section{Material and Methods}

\section{Theories}

\section{Ridge Regression}

Ridge regression, also called Tikhonov regularization, is a biased estimation regression method for linear data analysis which is essentially an improved least square estimation method [34]. When multicollinearity exists, the ridge regression method makes the estimation of the regression coefficient slightly biased through adding a non-negative factor $\mathrm{K}$ to the main diagonal element of the normalized matrix of the independent variable, then as a result, the stability of the estimator can be improved significantly, and the 
standard deviation of the regression system is smaller than the least squares estimator [35]. This paper uses the macro program Ridge Regression to do ridge regression in the SPSS software environment.

\section{IPAT Model and STIRPAT Model}

The IPAT model is also called Kaya identity, which can be obtained by decomposing the equation:

$$
C=P\left(\frac{G}{P}\right)\left(\frac{E}{G}\right)\left(\frac{C}{E}\right)=p g e c
$$

...where $C$ represents carbon emissions, $P$ is population, $G$ denotes GDP, $E$ denotes energy consumption; $G / P$ represents per capita GDP, $E / G$ denotes energy intensity, and $C / E$ denotes carbon emissions per unit of energy.

Dietz et al. (1994) established a stochastic model of STIRPAT to ameliorate the IPAT model:

$$
I=a P^{b} A^{c} T^{d} e
$$

...where $I, P, A$ and $T$ represent environmental stress, population size, affluence and technical level respectively; $a$ is the model coefficient; $b, c$ and $d$ represent the elasticity coefficient of population scale, affluence degree and technical level respectively; and $e$ is the model error term.

Compared with the IPAT model, the STIRPAT model has better expansibility. When assessing the environmental impact, multiple independent variables can be introduced to test the influence of each independent variable on environmental pressure.

Take the natural logarithm for formula (2) on both sides:

$$
\ln I=\ln a+b \ln \mathrm{P}+c \ln A+d \ln T+\ln e
$$

The standard regression coefficients of equation (3) reflect the extent and direction of the influence of the explanatory variables on the explanatory variables.

Based on the STIRPAT model, Huanan Li et al. [36] divided China's 30 provinces into five regions, and analyzed the carbon emissions of provinces from 1990 to 2010. The results show that population, GDP, technology, urbanization rate and industrial structure are the main factors affecting carbon emission. In this paper, we use the proportion of coal consumption to replace the carbon emissions per unit of energy $(\mathrm{C} / \mathrm{E})$ in formula (1), because the carbon emission per unit of energy is essentially the carbon emission coefficient, of which the value is basically fixed, and there will be errors in the ridge regression model when the variable is a constant. Considering that the carbon emission is mainly caused by coal consumption, the proportion of coal consumption is introduced as a variable. In addition, the urbanization rate and the proportion of service industry are added into the formula (3), thus a new STIRPAT model is obtained:

$$
\begin{aligned}
& \ln E M=a \ln P+b \ln A v G D P+c \ln E N E+ \\
& +d \ln P C+e \ln C I T Y+f \ln S E R V+\ln g
\end{aligned}
$$

...where $E M$ represents $\mathrm{CO}_{2}$ emissions (10,000 tons), $P$ is population (10,000 people), $A v G D P$ is GDP per capita (yuan/person), ENE is the energy intensity (tons of standard coal/10,000 yuan), $P C$ is the proportion of coal consumption, CITY is the urbanization rate, SERV is the proportion of service industry, and $g$ is a constant term [37-39].

\section{Genetic Algorithm}

Genetic algorithms (GA) is a new important branch of artificial intelligence, which is developed on the basis of Darwin's theory of evolution, and simulates the mechanism of biological evolution on the computer. It searches, calculates and solves problems according to the natural evolutionary rules such as survival of the fittest and so on. Moreover, it is an algorithm with strong global search ability and global optimization performance, which mainly includes operations of selection, crossover and mutation. Besides, it is robust, simple and versatile, which can be used in parallel distributed processing networks. Therefore, the genetic algorithm is used to narrow the search scope of BP neural networks in the training process by optimizing the weights and thresholds [40, 41].

\section{BP Neural Network}

Back propagation (BP) neural network is a threelayer or more network with no feedback and no interconnection, which consists of two processes: forward propagation of information and reverse propagation of error. In forward propagation, the input signal is input from the input layer, and then transmitted to the output layer after layer-by-layer processing of the hidden layer. If the actual output of the output layer does not match the expected value, the error back propagation process would be transferred. And the process of reverse propagation can reduce error through modifying the connection weight from the output layer to the input layer. The above two processes are cyclic. In general, the training process of BP neural network is the process of continuously adjusting connection weights, until the mean square error of the output is up to the required standard $[40,42]$.

\section{Optimization of BP Neural Network by Genetic Algorithm}

The algorithm flow of BP neural network based on genetic algorithm (GA-BP) is shown in Fig. 1. The calculation software is MATLAB R2015b in the operating environment of Windows 10. 


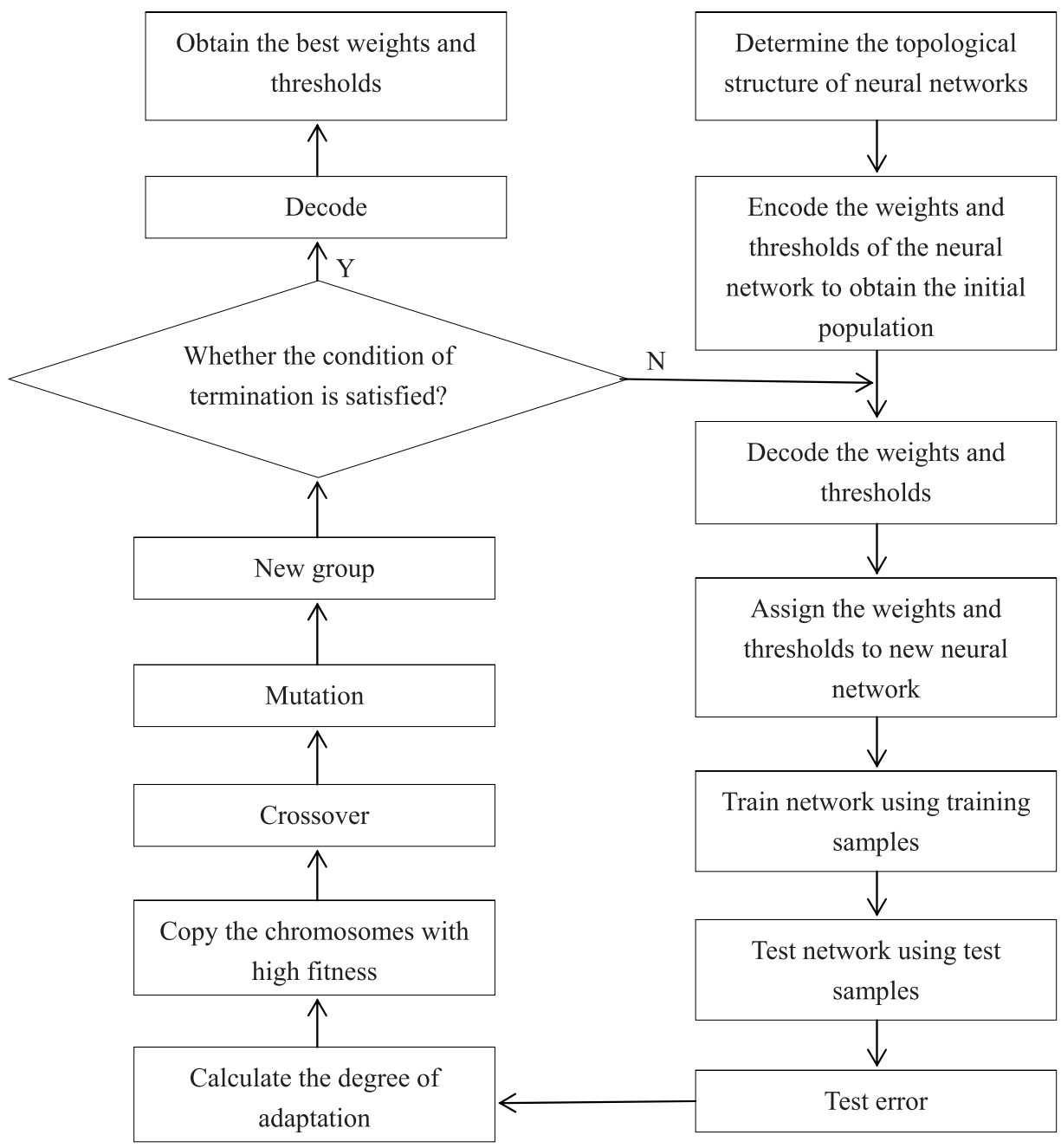

Fig. 1. Algorithm flow.

\section{Data}

\section{Data of STIRPAT Model}

The data of population, GDP, energy consumption, proportion of coal consumption, urbanization rate and the proportion of service industry in Hebei province from 1990 to 2015 are all from the Economic Yearbook of Hebei Province. The value of GDP is calculated at constant price in 2000. Carbon emissions are calculated by the following form:

$$
E M_{j}=\sum_{i} E_{i, j} \times \sigma_{i}
$$

...where $E M$ represents carbon emissions, $j$ represents years, $E$ represents energy, $i$ represent four kinds of energy consumption (coal, oil, natural gas and primary power), and $\sigma$ represents carbon emission coefficient.

\section{Data of GA-BP Model}

The data used in the GA-BP method are consistent with the STIRPAT model except for GDP per capita, which is replaced by GDP, namely population, GDP, energy intensity, coal consumption proportion, urbanization ratio, the proportion of service industry. The data used in the model during 1990-2015 can reference Table 4 (GDP can be calculated by "population $\times$ per capita GDP").

\section{$\mathrm{CO}_{2}$ Emission Coefficients of Coal, Oil and Natural Gas}

This paper collects two kinds of $\mathrm{CO}_{2}$ emission coefficients of coal, oil and natural gas. The first kind is obtained by calculation (Table $1, \mathrm{CO}_{2}$ emission coefficient I). The second is derived from Liang Wei's article [43] (Table 1, $\mathrm{CO}_{2}$ emission coefficient II). By comparison, the gap between the two kinds of coefficients is very small, and the second kind of coefficient is used in this paper.

\section{Carbon Emission Coefficient of Electrical Power}

"The 2010 China Low-Carbon Technology Fossil Fuel Grid Connected Power Generation Project 
Table 1. $\mathrm{CO}_{2}$ emission coefficients of coal, oil and natural gas.

\begin{tabular}{|c|c|c|c|c|c|c|}
\hline \multirow{2}{*}{ Energy } & \multirow{2}{*}{$\begin{array}{l}\text { Average low } \\
\text { calorific value }^{\text {a }} \\
\mathrm{kJ} / \mathrm{kg}\left(\mathrm{m}^{3}\right)\end{array}$} & \multirow{2}{*}{$\begin{array}{l}\text { Standard coal } \\
\text { coefficient }{ }^{\mathrm{a}} \\
\mathrm{kgce} / \mathrm{kg}\left(\mathrm{m}^{3}\right)\end{array}$} & \multirow{2}{*}{$\begin{array}{c}\text { Carbon content per unit } \\
\text { calorific value }{ }^{\mathrm{b}} \\
\text { Tons of carbon } / \mathrm{TJ}\end{array}$} & \multirow{2}{*}{$\begin{array}{l}\text { Carbon oxidation } \\
\text { rate }^{\mathrm{b}}\end{array}$} & \multicolumn{2}{|c|}{$\begin{array}{c}\mathrm{CO}_{2} \text { emission coefficient } \\
\mathrm{tCO}_{2} / \text { tce }\end{array}$} \\
\hline & & & & & I & II \\
\hline Raw coal & 20908 & 0.7143 & 26.37 & 0.94 & 2.6604 & 2.7689 \\
\hline Raw oil & 41816 & 1.4286 & 20.10 & 0.98 & 2.1141 & 2.1455 \\
\hline Natural gas & 38931 & 1.3300 & 15.30 & 0.99 & 1.6257 & 1.6420 \\
\hline
\end{tabular}

a Source: the general rules for calculation of comprehensive energy consumption (GB/T 2589-2008);

${ }^{\mathrm{b}}$ Source: the guidelines for the compilation of the greenhouse gas inventory at provincial level ([2011]1041).

Table 2. Power emission factors for all types of installed projects in $2008\left(\mathrm{tCO}_{2} / \mathrm{MWh}\right)$.

\begin{tabular}{|c|c|c|c|}
\hline Regional power grid & $600 \mathrm{MWe}$ & $660 \mathrm{MWe}$ & $1000 \mathrm{MWe}$ \\
\hline North China region & 0.8075 & 0.8065 & 0.7838 \\
\hline Northeast region & 0.7968 & 0.7968 & 0.7944 \\
\hline East China region & 0.7845 & 0.7825 & 0.7613 \\
\hline Central China region & 0.8044 & 0.7963 & 0.7963 \\
\hline Northwest Region & 0.8325 & 0.8311 & 0.8244 \\
\hline Southern Region & 0.8054 & 0.8008 & 0.8008 \\
\hline
\end{tabular}

Benchmark Emission Factor," published by the National Development and Reform Commission (NDRC), provides power emission factors applicable to various types of installed projects in 2008 (Table 2). As the data are all around 0.8 , the value of 0.8 is used as the power emission factor in this paper. Then according to "energy supply standard coal reference coefficient" (electric power is $0.1229 \mathrm{kgce} / \mathrm{kWh}$ ) provided by China Energy Statistics Yearbook, the $\mathrm{CO}_{2}$ emissions coefficient of electric power is calculated to be $0.0065 \mathrm{tCO}_{2} / \mathrm{tce}$.

\section{Model Construction}

\section{STIRPAT Model}

Based on the data of Hebei Province from 1990 to 2015 , we carry out a ridge regression analysis, and the standardized coefficients of each variable with $\mathrm{K}$ value are shown in Table 3, then Fig. 2 depicts the ridge trace.

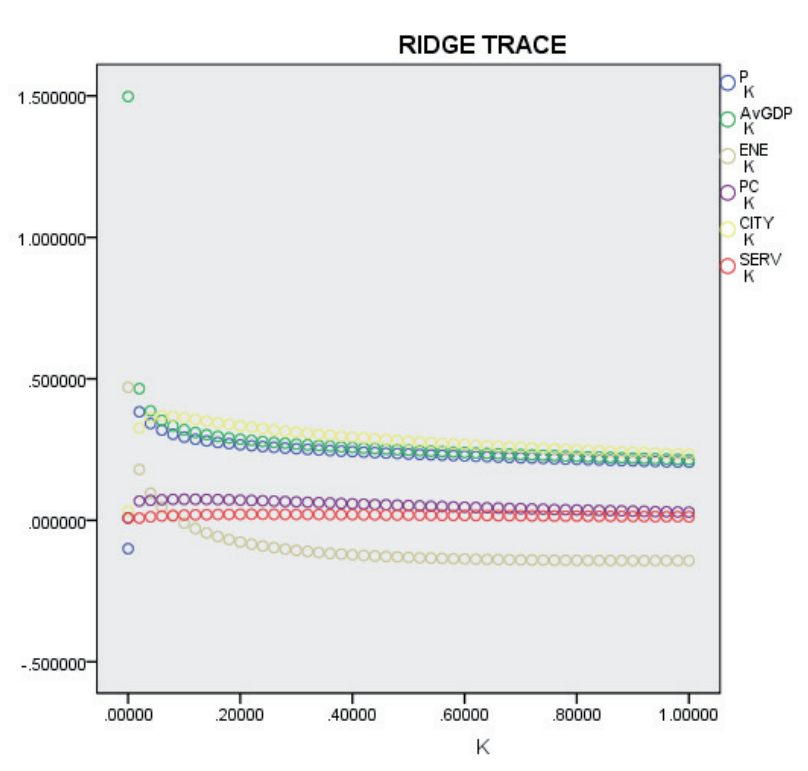

Fig. 2. Ridge trace.

When $\mathrm{K}$ is 0.4 , the coefficients of each variable tend to be stable, and the coefficient of determination is 0.9653 , which implies high goodness of fit. The variance analysis of the fitting results shows that the $\mathrm{F}$ test of the model is significant (Table 4). In addition, the results of nonstandard processing of coefficients are also calculated in Table 4. As a result, the equation corresponding to the STIRPAT model is obtained:

$\ln E M=2.7164 \ln P+0.1895 \ln A v G D P-0.2108 \ln E N E+$ $+2.7555 \ln P C+0.3442 \ln C I T Y+0.0788 \ln S E R V-27.0427$

Table 3. R-Square and beta coefficients for estimated values of K.

\begin{tabular}{|c|c|c|c|c|c|c|c|}
\hline $\mathrm{K}$ & $\mathrm{RSQ}$ & $\mathrm{P}$ & AvGDP & ENE & PC & CITY & SERV \\
\hline 0.0000 & 0.9999 & -0.0999 & 1.4930 & 0.4499 & 0.0208 & 0.0000 & -0.0012 \\
\hline 0.2500 & 0.9753 & 0.2694 & 0.2758 & -0.0669 & 0.0599 & 0.3163 & 0.0253 \\
\hline 0.5000 & 0.9587 & 0.2417 & 0.2458 & -0.1118 & 0.0619 & 0.2754 & 0.0198 \\
\hline 0.7500 & 0.9415 & 0.2238 & 0.2272 & -0.1260 & 0.0604 & 0.2492 & 0.0140 \\
\hline 1.0000 & 0.9233 & 0.2098 & 0.2128 & -0.1304 & 0.0579 & 0.2299 & 0.0094 \\
\hline
\end{tabular}


Table 4. Non-standardized coefficient.

\begin{tabular}{|c|c|c|c|c|c|c|c|}
\hline & $\mathrm{B}$ & $\mathrm{SE}(\mathrm{B})$ & Beta.std & $\mathrm{B} / \mathrm{SE}(\mathrm{B})$ & $\mathrm{t}$ & $\mathrm{F}$ value & Sig F \\
\hline $\mathrm{P}$ & 2.7164 & 0.1612 & 0.2508 & 16.8534 & 0.0000 & & \\
\hline AvGDP & 0.1895 & 0.0099 & 0.2555 & 19.2393 & 0.0000 & & \\
\hline ENE & -0.2108 & 0.0596 & -0.1000 & -3.5354 & 0.0028 & & \\
\hline PC & 2.7555 & 1.4803 & 0.0618 & 1.8614 & 0.0812 & & \\
\hline CITY & 0.34412 & 0.0295 & 0.2892 & 11.6686 & 0.0000 & & \\
\hline SERV & 0.0788 & 0.1183 & 0.0223 & 0.6665 & 0.5146 & & \\
\hline Constant & -27.0427 & 6.4554 & 0.0000 & -4.1891 & 0.0007 & & \\
\hline & & & & & & 74.2791 & 0.0000 \\
\hline
\end{tabular}

Formula (6) shows that the influence of the variables on $\mathrm{CO}_{2}$ emissions in the STIRPAT model from large to small is the proportion of coal consumption, population, urbanization rate, energy intensity, per capita GDP and the proportion of services.

a) Coal consumption proportion

The elasticity coefficient of coal consumption proportion to $\mathrm{CO}_{2}$ emissions in Hebei from 1990 to 2015 is 2.7555, of which the influence on $\mathrm{CO}_{2}$ emissions is the largest among all variables. Energy consumption in Hebei increased from 61.24 million TCE in 1990 to 193.95 million TCE in 2015, which had increased significantly since 2000. And during 1990-2015, the proportion of coal consumption in Hebei's energy consumption was about $90 \%$. Thus the effect of coal consumption proportion on $\mathrm{CO}_{2}$ emission is inevitable.

b) Population

The elasticity coefficient of population in Hebei is 2.7164, indicating that population has a positive effect on $\mathrm{CO}_{2}$ emissions. During 1990-2015, the population of Hebei increased from 61.59 million to 74.25 million, with a growth rate of $20.56 \%$. The growth of population made the corresponding growth of energy consumption a necessary condition to meet the basic needs of national survival and development, thus indirectly leading to the continuous rise of $\mathrm{CO}_{2}$ emissions.

c) Urbanization rate

In the model, the influence coefficient of urbanization rate is 0.3442 , which ranks third among all the variables. During 1990-2015, the urbanization rate of Hebei increased from $14.37 \%$ to $51.33 \%$, showing a fast urbanization process. However, the improvement of residents' living standards can promote the growth of living energy consumption, coupled with the production and consumption of energy mainly being in cities and towns. As a result, the process of urbanization leads to an increase in $\mathrm{CO}_{2}$ emissions.

d) Energy intensity

The influence coefficient of energy intensity in the model is -0.2108 , which is the only variable that has a negative impact on $\mathrm{CO}_{2}$ emissions, indicating that carbon emissions would increase with the decrease of energy intensity. The energy intensity of Hebei dropped from $4.0524 \mathrm{TCE} / 10,000$ yuan to $1.3121 \mathrm{TCE} / 10,000$ yuan during 1990-2015, which means that the growth rate of GDP in Hebei was greater than that of energy consumption. Although energy intensity is decreasing, energy consumption and GDP are growing, thus carbon emission increases.

e) Per capita GDP

As for per capita GDP, its impact on $\mathrm{CO}_{2}$ emissions in Hebei is not as significant as other variables, but also plays a positive role in promoting $\mathrm{CO}_{2}$ emissions. Over the past two decades, Hebei's economy developed rapidly, and its per capita GDP increased from 2,454 yuan to 30,174 yuan during 1990-2015 - an increase by more than 11 times. Rapid growth of the economy plays a great role in promoting energy demand and $\mathrm{CO}_{2}$ emissions. Nevertheless, as the economy and wealth grow, more capital will be spent on $R \& D$ as well as the introduction of low-carbon technologies, which leads to the improvement of efficiency of energy utilization, thus energy consumption and carbon emissions per unit output are depressed.

f) Proportion of service industry

The proportion of service industry has the least impact on $\mathrm{CO}_{2}$ emissions in Hebei, which is only 0.0788. China's economy was not yet developed in the 1990 s, when the proportion of the service industry was relatively large. And then with the development of industry, the proportion of the service industry declined. In recent years, China's industry has developed very well, and faced adjustment and reformation on the industrial structure. According to the $13^{\text {th }}$ Five-Year Plan, by 2020 the added value of service industry in Hebei should account for about 45\% of GDP. From another point of view, the small proportion of service industry reflects that the impact of industrial structure on carbon emissions is mainly concentrated on other industries. Since the proportion of the service industry will continue to rise in the coming years, it is of some significance to study the service industry. 


\section{GA-BP Model}

The differences in magnitude of the vectors in the raw data sample are large, which is not conducive to the training and calculation of the network, therefore it is necessary to normalize the raw data samples. In this paper, the "mapminmax" function is used to normalize the input and output data, so that the processed data are evenly distributed in the range of $[-1,1]$, and the syntax format is "[y1, PS] = mapminmax(x1)". When another set of data needs to do the same normalization process, the syntax format is " $y 2$ = mapminmax ('apply', $\mathrm{x} 2$, PS)". The corresponding transformation formula is as follows:

$$
X=\frac{X-X_{\min }}{X_{\max }-X_{\min }}
$$

...where $X$ represents the original sample data, andrepresents the normalized sample data. After the BP neural network training is finished, the function "mapminmax" is used to reverse normalization, and the syntax format is " $\mathrm{x} 1$ '= mapminmax ('reverse', yl, PS)".

a) Initialization parameter setting

In this paper, the training method of BP neural network is a descent method with momentum gradient, the training function is "traingdm," and the performance function is "MSE." Besides, the learning rate is set to 0.1 , maximum training time is set to 1000 , and the target error of training is 0.0001 ; other parameters are default values.

b) The number of hidden layer nodes

The BP neural network used in this paper is a threelayer structure neural network. According to the general rules of experience, the number of nodes in a single hidden layer is determined by the following formula:

$$
b=\sqrt{a+c}+m
$$

...where $a$ represents the number of nodes in the input layer, $c$ represents the number of nodes in the output layer, $b$ represents the number of nodes in the hidden layer, and $m$ represents an integer in the range of 1 to 10 . In this paper, the input layer has 6 nodes, and the output layer has 1 . The number of nodes in the hidden layer is set to 9 .

c) The setting of transfer function

The transfer function of the hidden layer uses the sigmoid tangent function "tansig," and the transfer function of the output layer is the "purelin" function.

d) Parameter setting of genetic algorithm

In the genetic algorithm, the maximum genetic algebra is set to 30 , the binary number of variables is 10 , the generation gap is set to 0.95 , and the crossover probability is set to 0.7 , the mutation probability is 0.01 .

\section{Model Checking}

Using the STIRPAT model and GA-BP model, the $\mathrm{CO}_{2}$ emissions of Hebei from 1990 to 2015 are predicted, which are compared with the raw data. The results are shown in Fig. 3.

In the STIRPAT model, the fluctuation of raw data is large, while the prediction data is relatively stable. Though there are partial deviations (the maximum error is as high as $20 \%$ ) in the fitting result, most of the errors are within $10 \%$, and the prediction errors of the data tend to 0 since 2014 . Thus the overall stability of the STIRPAT model is good.

In the GA-BP model, we use the data of 21 years from 1990 to 2010 as the training data, and the data from 2011 to 2015 as test data. The error evolution diagram of GA optimization process is shown in Fig. 4. It can be seen from Fig. 3 that the prediction errors of the GA-BP network are basically within $\pm 10 \%$, and most of the errors are at the interval of $[-4 \%, 2 \%]$; therefore the training result is good.

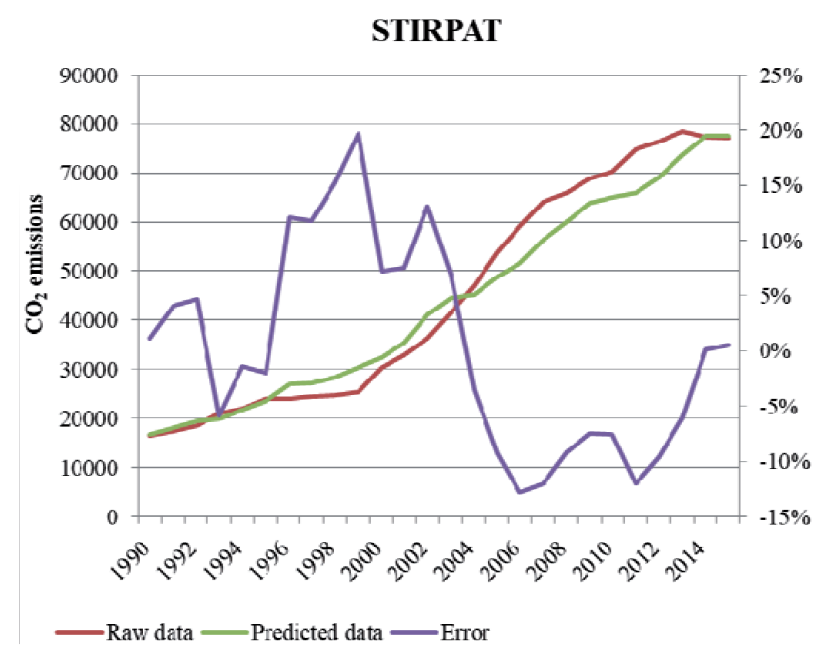

GA-BP

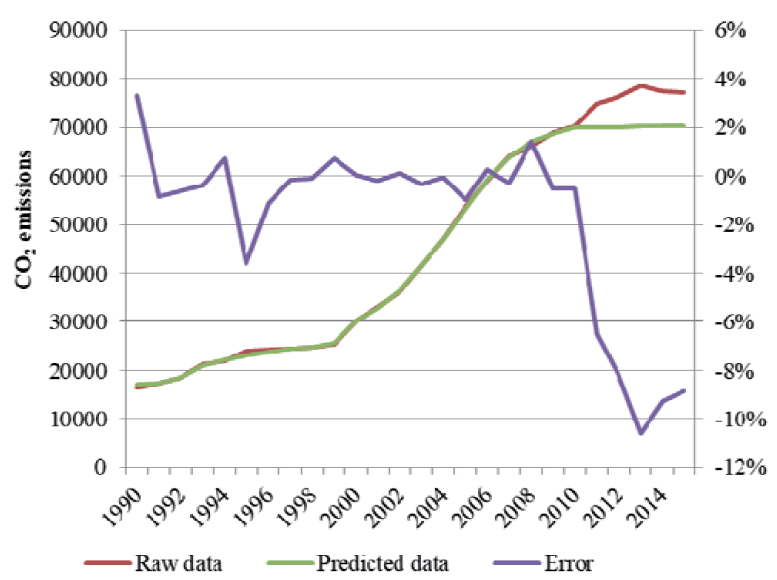

Fig. 3. Comparison of $\mathrm{CO}_{2}$ emissions between predicted data and raw data during 1990-2015. 


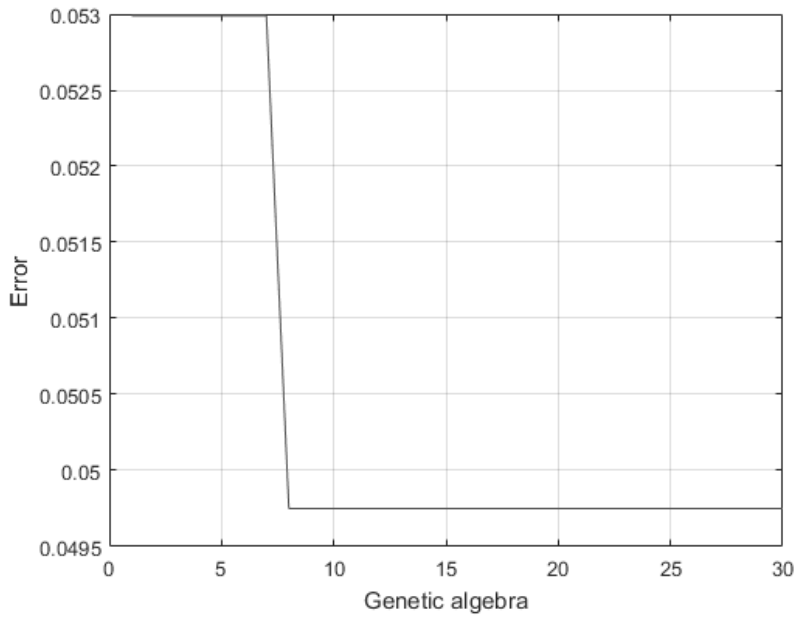

Fig. 4. Error evolution diagram of GA optimization process.

\section{Scenarios Setting}

\section{Population}

It can be seen from formula (6) that population has a significant impact on the carbon emissions of Hebei. According to the Provincial Health Committee and Provincial Family Planning Commission, affected by the decline in birth rate and the rise in mortality levels, the growth rate of the province's population will decline rapidly during the $13^{\text {th }}$ Five-Year; however, the population will still present a growth trend of inertia. It is estimated that the population of Hebei will peak at 77 million around 2025, and then begin to decrease slowly [44]. In 2015, the population of Hebei was 74.25 million, thus the average annual growth rate of Hebei's population during 2016-2025 would be $0.364 \%$ with the supposition that the population would peak at 77 million in 2025 (Scenario B). Besides, this paper assumes that scenarios $\mathrm{A}$ and $\mathrm{C}$ reached the population peak of 77 million in 2028 and 2022, respectively. And the average annual deceleration rates after the peak years are all set to $0.2 \%$ in the three scenarios (Table 5).

\section{$G D P$}

The $13^{\text {th }}$ Five-Year Plan of Hebei pointed out that Hebei is to increase the annual GDP by about 7\%, and break through the GDP target of 4 trillion yuan by 2020 (at the current price). Accordingly, the growth rates of GDP with low, medium, and high speeds in Hebei from 2016 to 2035 are formulated (Table 5). The per capita GDP $(A v G D P)$ required by the STIRPAT model is calculated by GDP/ Population.

\section{Energy Consumption}

According to Hebei's $13^{\text {th }}$ Five-Year Plan Greenhouse Gas Emission Control Project, the province is going
Table 5. Scenario settings.

\begin{tabular}{|c|c|c|c|c|}
\hline Scenarios & & Year & \multicolumn{2}{|c|}{ Growth rate $(\%)$} \\
\hline \multirow{7}{*}{ Population } & \multirow{2}{*}{ A } & $2016-2028$ & \multicolumn{2}{|c|}{0.280} \\
\hline & & 2029-2030 & -0.20 & \\
\hline & \multirow{2}{*}{ B } & $2016-2025$ & \multicolumn{2}{|c|}{0.364} \\
\hline & & $2026-2030$ & \multicolumn{2}{|c|}{-0.200} \\
\hline & \multirow{2}{*}{$\mathrm{C}$} & $2016-2022$ & \multicolumn{2}{|c|}{0.521} \\
\hline & & $2023-2030$ & \multicolumn{2}{|c|}{-0.200} \\
\hline & Year & Low & Medium & High \\
\hline \multirow{4}{*}{ GDP } & 2016-2020 & 6.5 & 7.0 & 7.5 \\
\hline & $2021-2025$ & 6.0 & 6.5 & 7.0 \\
\hline & $2026-2030$ & 5.5 & 6.0 & 6.5 \\
\hline & $2031-2035$ & 4.5 & 5.0 & 5.5 \\
\hline $\begin{array}{l}\text { Total energy } \\
\text { consumption }\end{array}$ & 2016-2035 & 2.0 & 2.5 & 3.0 \\
\hline $\begin{array}{c}\text { Coal } \\
\text { consumption } \\
\text { proportion }\end{array}$ & $2016-2035$ & -0.5 & -1.0 & -1.5 \\
\hline \multirow{2}{*}{$\begin{array}{c}\text { Urbanization } \\
\text { rate }\end{array}$} & $2016-2020$ & 2.5 & 3.0 & 3.5 \\
\hline & $2021-2035$ & 1.0 & 1.5 & 2.0 \\
\hline $\begin{array}{l}\text { Proportion of } \\
\text { service industry }\end{array}$ & $2016-2035$ & 2.0 & 2.5 & 3.0 \\
\hline
\end{tabular}

to build a low-carbon energy system, and strengthen the control of energy consumption and intensity. Hebei ensured that, by 2020, the energy consumption of the province would be controlled within 327.85 million tons of standard coal and the energy consumption of per 10,000 yuan of GDP would decrease by $17 \%$ compared with 2015. Assuming that the energy consumption of Hebei in 2020 would be 327.85 million tons of standard coal, the corresponding average annual growth rate of energy consumption from 2016 to 2020 is calculated at $2.2 \%$. Accordingly, the growth rates of energy consumption with low, medium, and high speeds in Hebei during 2016-2035 are formulated (Table 5). The energy intensity (ENE) required by the STIRPAT model is calculated by Energy consumption/ GDP.

\section{Coal Consumption Proportion}

According to Hebei's $13^{\text {th }}$ Five-Year Plan Greenhouse Gas Emission Control Project, the province should greatly reduce coal consumption through suppressing industrial coal combustion and governing decentralized coal in order to ensure that the total amount of coal consumption in 2017 would decrease by 40 million tons compared to 2012. Thus the average annual decrease rate of coal consumption in Hebei from 2012 to 2017 is $3 \%$ (Table 5). 
Table 6. Final scenarios setting.

\begin{tabular}{|c|c|c|c|c|c|c|}
\hline Population & $\mathrm{GDP}$ & $\begin{array}{c}\text { Energy con- } \\
\text { sumption }\end{array}$ & $\begin{array}{c}\text { Coal consump- } \\
\text { tion proportion }\end{array}$ & $\begin{array}{c}\text { Urbanization } \\
\text { rate }\end{array}$ & $\begin{array}{c}\text { Proportion of } \\
\text { service industry }\end{array}$ & Sub-scenarios \\
\hline \multirow{4}{*}{$\mathrm{A}$} & $\mathrm{G}_{1}$ & $\mathrm{E}_{1}$ & $\mathrm{CO}_{1}$ & $\mathrm{U}_{1}$ & $\mathrm{~S}_{1}$ & $\mathrm{~A} 1$ \\
\cline { 2 - 7 } & $\mathrm{G}_{\mathrm{m}}$ & $\mathrm{E}_{\mathrm{m}}$ & $\mathrm{CO}_{\mathrm{m}}$ & $\mathrm{U}_{\mathrm{m}}$ & $\mathrm{S}_{\mathrm{m}}$ & $\mathrm{A} 2$ \\
\cline { 2 - 7 } & $\mathrm{G}_{\mathrm{h}}$ & $\mathrm{E}_{\mathrm{h}}$ & $\mathrm{CO}_{\mathrm{h}}$ & $\mathrm{U}_{\mathrm{h}}$ & $\mathrm{S}_{\mathrm{h}}$ & $\mathrm{A} 3$ \\
\hline \multirow{3}{*}{$\mathrm{B}$} & $\mathrm{G}_{1}$ & $\mathrm{E}_{1}$ & $\mathrm{CO}_{1}$ & $\mathrm{U}_{1}$ & $\mathrm{~S}_{1}$ & $\mathrm{~B} 1$ \\
\cline { 2 - 7 } & $\mathrm{G}_{\mathrm{m}}$ & $\mathrm{E}_{\mathrm{m}}$ & $\mathrm{CO}_{\mathrm{m}}$ & $\mathrm{U}_{\mathrm{m}}$ & $\mathrm{S}_{\mathrm{m}}$ & $\mathrm{B} 2$ \\
\cline { 2 - 7 } & $\mathrm{G}_{\mathrm{h}}$ & $\mathrm{E}_{\mathrm{h}}$ & $\mathrm{CO}_{\mathrm{h}}$ & $\mathrm{U}_{\mathrm{h}}$ & $\mathrm{S}_{\mathrm{h}}$ & $\mathrm{B} 3$ \\
\hline \multirow{3}{*}{$\mathrm{C}$} & $\mathrm{G}_{1}$ & $\mathrm{E}_{1}$ & $\mathrm{CO}_{1}$ & $\mathrm{U}_{1}$ & $\mathrm{~S}_{1}$ & $\mathrm{C} 1$ \\
\cline { 2 - 7 } & $\mathrm{G}_{\mathrm{m}}$ & $\mathrm{E}_{\mathrm{m}}$ & $\mathrm{CO}_{\mathrm{m}}$ & $\mathrm{U}_{\mathrm{m}}$ & $\mathrm{S}_{\mathrm{m}}$ & $\mathrm{C} 2$ \\
\cline { 2 - 7 } & $\mathrm{G}_{\mathrm{h}}$ & $\mathrm{E}_{\mathrm{h}}$ & $\mathrm{CO}_{\mathrm{h}}$ & $\mathrm{U}_{\mathrm{h}}$ & $\mathrm{S}_{\mathrm{h}}$ & $\mathrm{C} 3$ \\
\hline
\end{tabular}

\section{Urbanization Rate}

According to the Outline of Collaborative Development of Beijing, Tianjin and Hebei Province, the urbanization rate in Hebei will reach about $60 \%$ for permanent residents. Thus the average annual growth rate of urbanization rate in Hebei from 2016 to 2020 is $3.17 \%$. According to this, the urbanization rates with low, medium, high speeds in Hebei province from 2016 to 2035 are formulated (Table 5).

\section{Proportion of Service Industry}

According to Hebei's $13^{\text {th }}$ Five-Year Plan Greenhouse Gas Emission Control Project, by 2020 the added value of services should account for about $45 \%$ of GDP. Thus the average annual growth rate of proportion of service industry in Hebei from 2016 to 2020 is 2.28\%. According to this, the proportions of service industry from 2016 to 2035 of low, medium, abd high speeds are formulated (Table 5).

\section{Final Scenarios}

As the population has a significant impact on carbon emissions and differs from other variables, it was not explicitly put forward mandatory requirements in any published government reports, therefore this paper takes population as the parental scene, and uses GDP, energy consumption, coal consumption proportion, urbanization rate and the proportion of service industry as sub-scenarios. The final scenario settings are shown in Table 6.

\section{Results and Discussion}

\section{STRPAT Model}

The forecast results of carbon emissions from 2016 to 2035 in Hebei by STIRPAT model are shown in
Fig. 5. As shown in the figure, the results of 9 scenarios present three different trends affected by the sub-scenes (GDP, energy consumption, coal consumption proportion, urbanization rate and the proportion of service industry). Among them, carbon emissions of scenarios A1, B1, C1 are the highest, followed by A2, B2, C2, and finally A3, B3, C3. Each trend is influenced by the parental scenario (population), which makes the carbon emission arrangement appear as $\mathrm{A}>\mathrm{B}>\mathrm{C}$. However, in the initial stage, the faster the population growths, the faster carbon emission increases, and as a result the carbon emissions are $\mathrm{C}>\mathrm{B}>\mathrm{A}$ before 2022, and then there is a shift during 2022-2028.

The three scenarios of $\mathrm{A} 1, \mathrm{~B} 1$ and $\mathrm{C} 1$ achieve carbon emission peaks in 2030, of which the peak years are not affected by different population scenarios, and their carbon emission peaks are 1,007.2901 million tons, 992.0801 million tons and 977.2396 million tons, respectively. In scenario A2, the peak year is 2028, and the carbon emission peak is 868.7079 million tons; in scenario B2, the peak year of carbon emissions is 2025, with corresponding carbon emissions of 874.2155 million tons; and as for scenario $\mathrm{C} 2$, the carbon emission peak is 875.9241 million tons in 2022 . The peak years of carbon emission in scenarios A3 and B3 are both 2020, with corresponding carbon emissions at 784.1635 million tons and 791.3301 million tons, respectively; and in scenario $\mathrm{C} 3$ the carbon emission peak is 810.7182 million tons in 2022 .

It can be drawn from Fig. 5 that after reaching the carbon emissions peak, the decreases of carbon emissions in low sub-scenes (A1, B1, C1) are very slow, of which the trends are almost horizontal; carbon emissions of medium sub-scenes (A2, B2, C2) decrease relatively faster; and the descents of carbon emission of high sub-scenes (A3, B3, C3) are the fastest. Therefore, the earlier the carbon emission peak is achieved, the more conducive it is to the realization of carbon emission reduction. 


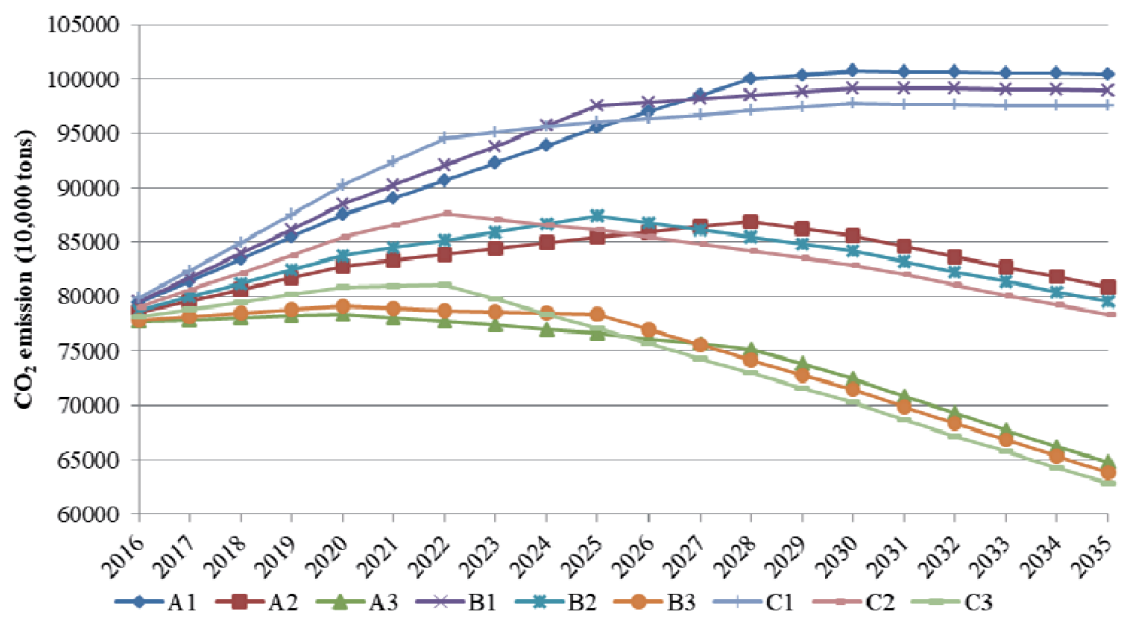

Fig. 5. Carbon emission trend curve of Hebei Province from 2016 to 2035 (STIRPAT model).

\section{GA-BP Model}

The forecast results of carbon emissions from 2016 to 2035 in Hebei by GA-BP model are shown in Fig. 6. It can be seen from Fig. 6 that results are relatively stable. The maximum carbon emissions peak is reached in 2022 under the $\mathrm{C} 1$ scenario, which is 702.8114 million tons; and the minimum carbon emissions peak is reached in 2016 under the A3 scenario with corresponding carbon emissions of 702.7465 million tons. The disparity is only 64.9 thousand tons.

The peak years of carbon emissions are as follows: Scenarios A1, A2, and A3 are in 2028, with corresponding carbon emissions peaks of 702.8088 million tons, 702.775 million tons and 702.7465 million tons, respectively; Scenarios B1, B2, and B3 are in 2025, with corresponding peak values of 702.8109 million tons, 702.7818 million tons and 702.7518 million tons, respectively; and scenarios C1, C2, and C3 are in 2022, with corresponding carbon emissions peaks of 702.8114 million tons, 702.7908 million tons and 702.7663 million tons, respectively. It is not difficult to find that the peak years of the predicted carbon emissions are in line with the peak years of population in all scenarios, which shows that the population has a significant impact on carbon emissions in this model. In addition, on the condition of same sub-scenarios (GDP, energy intensity, coal consumption proportion, urbanization rate, proportions of service industry), the higher the parental scenario (population) is, the greater the carbon emissions will be, namely $\mathrm{C}>\mathrm{B}>\mathrm{A}$.

\section{Comparing Results between STIRPAT and GA-BP Models}

(1) Similarities

From the above analysis, it can be seen that the years of carbon emission peaks are all in 2030 or before 2030; therefore it is not difficult for Hebei to achieve the peak target of carbon emissions in 2030. And the results of the two models are significantly affected by demographic factors, especially the GA-BP model, of which the peak years of carbon

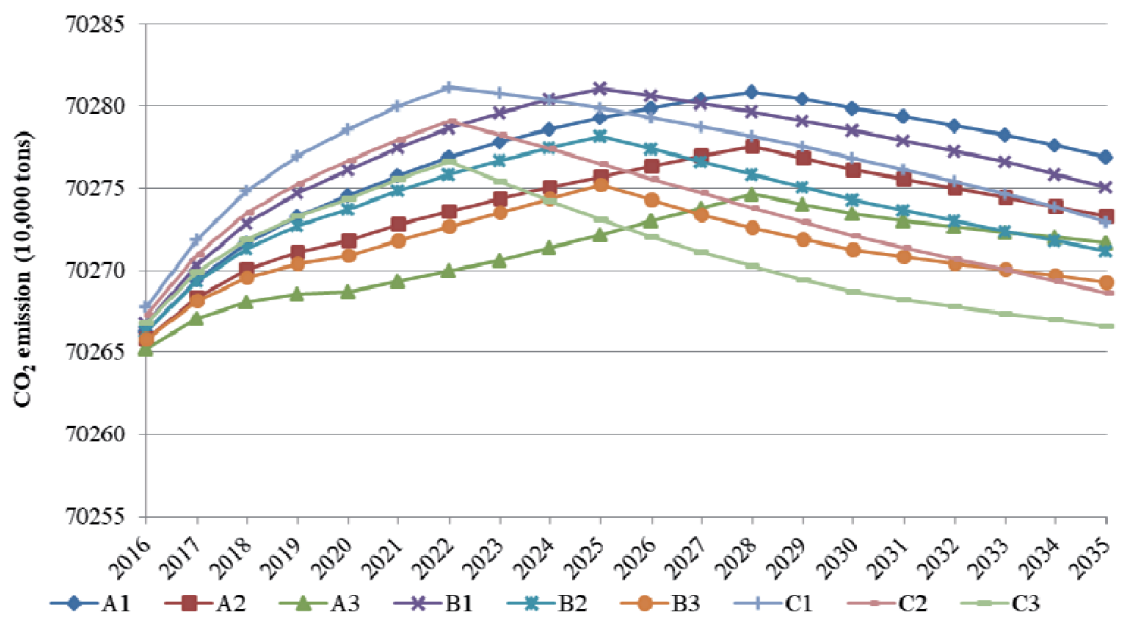

Fig. 6. Carbon emissions trend curve of Hebei Province from 2016 to 2035 (GA-BP model). 
emission are consistent with the peak years of population. In addition, affected by sub-scenarios, the carbon emission peaks would reach later in lower sub scenarios.

(2) Differences

The values of carbon emission peak predicted by the STIRPAT model vary from 784.1635 million tons to $1,007.2901$ million tons, with corresponding peak years from 2022 to 2030 . And the results present obvious classification phenomenon affected by subscenarios. Influenced by a demographic factor, there is a shift of the rankings of carbon emission during 2022-2028.

The results of the GA-BP model are more stable, and the values of carbon emission peaks vary from 702.7465 million tons to 702.8144 million tons. The carbon emission curves are more concentrated and there's no obvious classification phenomenon that can be found in the STIRPAT model. Besides, the predicted peak years are only three - 2022, 2025 and 2028 - in line with the population peak years.

The comparison between the predicted data and raw data of carbon emissions from 1990 to 2015 (Fig. 3) shows that the GA-BP model has a better fitting effect, with higher accuracy of carbon emission prediction. However, the GA-BP model is greatly affected by demographic factor, which may reduce the reliability of the peak years predicted by the model. Therefore, the STIRPAT model should be more valuable in the predicted peak years of carbon emissions.

\section{Conclusions}

Based on the analysis of the status quo of economic and social development in Hebei Province, this paper uses population, GDP, energy consumption, coal consumption proportion, urbanization rate and the proportion of service industry in Hebei as the analysis variables, and applies the two models of STIRPAT and GA-BP to analyze the data of the province during 19902015. The carbon emission peaks of Hebei from 2016 to 2035 under nine scenarios are predicted.

Firstly, the studied driving factors of carbon emissions in Hebei are population, GDP, energy consumption, coal consumption proportion, urbanization rate and service industry proportion. Ridge regression analysis shows that population and the proportion of coal consumption are the two most important factors, and the proportion of service industry has the least impact on carbon emissions. Secondly, this paper constructs nine scenarios, taking the population as the parental scene, and the remaining factors as sub-scenarios. Thus, nine scenarios are obtained: A1, A2, A3; B1, B2, B3; C1, C2, C3. Thirdly, this paper uses the STIRPAT model to predict the carbon emissions of Hebei from 1990 to 2015 with population, per capita GDP, energy intensity, coal consumption proportion, urbanization rate and the proportion of service industry as independent variables. By comparing with the raw data, we found that the STIRPAT model has a good prediction effect. Influenced by the scenarios, the predicted results of carbon emissions vary greatly, but the peak years of carbon emissions in Hebei are all in or before 2030. Fourthly, taking population, GDP, energy intensity, coal consumption proportion, urbanization rate and service industry proportion as independent variables, the GABP model has a fine fitting effect and it's more stable than the STIRPAT model. The peak years of carbon emissions in nine scenarios are consistent with the peak years of population; therefore, the model's reliability is reduced. Nevertheless the carbon emission predicted by the GA-BP model is more stable and accurate than the STIRPAT model.

It is hence concluded that the earlier the carbon emissions peak is achieved, the more conducive to the realization of carbon emission reduction. 1) Population is an important factor that influences carbon emission. With the implementation of the two children policy, population could keep increasing for a short term, so for the purposes of controlling carbon emissions, the increase of population should be suppressed properly. 2) Coal consumption proportion is another key factor. Attention should be paid to alternative coal work and speed up the pace of energy transformation, which also contributes to the governance of smog. We need to encourage investment in new high-technology industries such as clean energy and environmental technology, as well as use more clean energy and less coal and oil. 3) While promoting the urbanization process, we should also pay attention to carbon emission reduction. We should intensify efforts to prevent and control environmental pollution, do a good job in environmental protection in key cities, and strengthen environmental protection and construction in small towns.

\section{Acknowledgements}

This study is supported by the National Social Science Foundation of China (NSSFC) (Grant No.15BGL145).

\section{Conflict of Interest}

The authors declare that there is no conflict of interest regarding the publication of this paper.

\section{References}

1. China Energy editorial department: China-U.S. Joint Presidential Statement on Climate Change. Energy of China. 36, 1, 2014.

2. Commission $\mathrm{CDaR}$. Outline of the thirteenth five year plan for China's national economic and social development. 2016. 
3. SHI Y.P., YAN F.G. Structural decomposition analysis of carbon emission intensity in Hebei province based on MMIA. Science Technology and Industry. 16, 121, 2016.

4. WANG P., DAI H.C., REN S.Y., ZHAO D.Q., MASUI T. Achieving Copenhagen target through carbon emission trading: Economic impacts assessment in Guangdong Province of China. Energy 79, 212, 2015.

5. LU Q., YANG H., HUANG X., CHUAI X., WU C. Multisectoral decomposition in decoupling industrial growth from carbon emissions in the developed Jiangsu Province, China. Energy 82, 414, 2015.

6. ZHANG N., WANG B., LIU Z. Carbon emissions dynamics, efficiency gains, and technological innovation in China's industrial sectors. Energy 99, 10, 2016.

7. MONDAL M.K., BALSORA H.K., VARSHNEY P. Progress and trends in $\mathrm{CO}_{2}$ capture/separation technologies: A review. Energy 46, 431, 2012.

8. LAJUNEN A., LIPMAN T. Lifecycle cost assessment and carbon dioxide emissions of diesel, natural gas, hybrid electric, fuel cell hybrid and electric transit buses. Energy 106, 329, 2016.

9. XU B., LIN B. Carbon dioxide emissions reduction in China's transport sector: A dynamic VAR (vector autoregression) approach. Energy 83, 486, 2015.

10. TANG C.F., TAN B.W. The impact of energy consumption, income and foreign direct investment on carbon dioxide emissions in Vietnam. Energy 79, 447, 2015.

11. ÖZBUĞDAY F.C., ERBAS B.C. How effective are energy efficiency and renewable energy in curbing $\mathrm{CO}_{2}$ emissions in the long run? A heterogeneous panel data analysis. Energy 82, 734, 2015.

12. KARMELLOS M., KOPIDOU D., DIAKOULAKI D. A decomposition analysis of the driving factors of $\mathrm{CO}_{2}$ (Carbon dioxide) emissions from the power sector in the European Union countries. Energy 94, 680, 2016.

13. LIN S., WANG S., MARINOVA D., ZHAO D., HONG J. Impacts of urbanization and real economic development on $\mathrm{CO}_{2}$ emissions in non-high income countries: Empirical research based on the extended stirpat model. Journal of Cleaner Production. 166, 952, 2017.

14. WANG Z., YIN F., ZHANG Y., ZHANG X. An empirical research on the influencing factors of regional $\mathrm{CO}_{2}$ emissions: Evidence from Beijing city, China. Appl. Energ. 100, 277, 2012

15. WANG P., WU W., ZHU B., WEI Y. Examining the impact factors of energy-related $\mathrm{CO}_{2}$ emissions using the STIRPAT model in Guangdong Province, China. Appl. Energ. 106, 65, 2013.

16. WANG Y., KANG Y., WANG J., XU L. Panel estimation for the impacts of population-related factors on $\mathrm{CO}_{2}$ emissions: A regional analysis in China. Ecological Indicators. 78, 322, 2017.

17. HUBACEK K., FENG K., CHEN B. Changing Lifestyles Towards a Low Carbon Economy: An IPAT Analysis for China. Energies. 5, 22, 2011.

18. XIA C., LI Y., YE Y., SHI Z., LIU J. Decomposed Driving Factors of Carbon Emissions and Scenario Analyses of Low-Carbon Transformation in 2020 and 2030 for Zhejiang Province. Energies. 10, 1747, 2017.

19. ZHAO C., CHEN B., HAYAT T., ALSAEDI A., AHMAD $B$. Driving force analysis of water footprint change based on extended STIRPAT model: Evidence from the Chinese agricultural sector. Ecological Indicators. 47, 43, 2014.

20. SHAHBAZ M., CHAUDHARY A.R., OZTURK I. Does urbanization cause increasing energy demand in Pakistan?
Empirical evidence from STIRPAT model. Energy 122, 83, 2017.

21. XU L., CHEN N., CHEN Z. Will China make a difference in its carbon intensity reduction targets by 2020 and 2030 ? Appl. Energ. 203, 874, 2017.

22. SARSHAR J., MOOSAPOUR S.S., JOORABIAN M. Multi-objective energy management of a micro-grid considering uncertainty in wind power forecasting. Energy 139, 680, 2017

23. KADHEM A.A., WAHAB N., ARIS I., JASNI J., ABDALLA A. Advanced Wind Speed Prediction Model Based on a Combination of Weibull Distribution and an Artificial Neural Network. Energies. 10, 1744, 2017.

24. YUCE B., MOURSHED M., REZGUI Y. A Smart Forecasting Approach to District Energy Management. Energies. 10, 1073, 2017.

25. LE VAN S.C., B.H. Applicability of an Artificial Neural Network for Predicting Water-Alternating- $\mathrm{CO}_{2}$ Performance. Energies. 10, 842, 2017.

26. TAHERI-RAD A., KHOJASTEHPOUR M., ROHANI A., KHORAMDEL S., NIKKHAH A. Energy flow modeling and predicting the yield of Iranian paddy cultivars using artificial neural networks. Energy 135, 405, 2017.

27. VÁSQUEZ N., MAGÁN C., OBLITAS J., CHUQUIZUTA T., AVILA-GEORGE H., CASTRO W. Comparison between artificial neural network and partial least squares regression models for hardness modeling during the ripening process of Swiss-type cheese using spectral profiles. Journal of Food Engineering. 219, 8, 2018.

28. GARCIA-SEGURA R., VÁZQUEZ CASTILLO J., MARTELL-CHAVEZ F., LONGORIA-GANDARA O., ORTEGÓN AGUILAR J. Electric Arc Furnace Modeling with Artificial Neural Networks and Arc Length with Variable Voltage Gradient. Energies. 10, 1424, 2017.

29. TANZIFI M., YARAKI M.T., KIADEHI A.D., HOSSEINI S.H., OLAZAR M., BHARTI A.K., AGARWAL S., GUPTA V.K., KAZEMI A. Adsorption of Amido Black $10 \mathrm{~B}$ from aqueous solution using polyaniline/ $\mathrm{SiO}_{2}$ nanocomposite: Experimental investigation and artificial neural network modeling. J Colloid Interface Sci. 510, 246, 2018.

30. MORSE L., SHARIF KHODAEI Z., ALIABADI M.H Reliability based impact localization in composite panels using Bayesian updating and the Kalman filter. Mechanical Systems and Signal Processing. 99, 107, 2018

31. SOLTANALI H., NIKKHAH A., ROHANI A. Energy audit of Iranian kiwifruit production using intelligent systems. Energy 139, 646, 2017.

32. RASHIDI M.M., AGHAGOLI A., RAOOFI R. Thermodynamic analysis of the ejector refrigeration ycle using the artificial neural network. Energy 129, 201, 2017.

33. FAZLI SHAHRI H.R., MAHDAVINEJAD R. Prediction of temperature and HAZ in thermal-based processes with Gaussian heat source by a hybrid GA-ANN model. Optics \& Laser Technology. 99, 363, 2018.

34. HOERL A.E., KENNARD R.W. Ridge regression: biased estimation for nonorthogonal problems: American Society for Quality Control and American Statistical Association. 2000.

35. LI B.R. Econometrics (Second Edition). China Mechine Press. 2015

36. LI H., MU H., ZHANG M., GUI S. Analysis of regional difference on impact factors of China's energy - Related $\mathrm{CO}_{2}$ emissions. Energy 39, 319, 2012. 
37. DU Q., CHEN Q., LU N. Forecast of China's carbon emissions based on modified IPAT model. Acta Scientiae Circumstantiae. 32, 2294, 2012.

38. SONG X.H., ZHANG Y.F., WANG Y.M., FENG Y.C. Analysis of impacts of demographic factors on carbon emissions based on the IPAT model. Research of Environmental Sciences. 25, 109, 2012.

39. YANG M., CHEN K. Influencing factors of carbon emissions in Hebei province based on the STIRPAT and Decoupling Models. Journal of Northeastern University (Natural Science). 38, 300, 2017.

40. GAO Y.M., ZHANG R.J. Analysis of house price prediction based on Genetic Algorithm and BP Neural Network. Computer Egineering. 40, 187, 2014.
41. ZANG D.W., LU B.H., ZHU C.F., LU J.Y., LIU H., ZUO J. Study of GA-BP Neural Network for water requirement prediction based on Gray Correlation Analysis. Water Resources and Power. 39, 2015.

42. JI G.Y. Application of BP neural network model in the prediction of China's carbon emissions based on Grey Correlation analysis. 44, 243, 2014.

43. LIANG W. Study of double dividend of environmental tax based on CGE model - A case study of Shandong province. Tianjin University. 2013.

44. SUN M.X. Hebei News Network [http://hebei.hebnews. cn/2014-07/11/content_4033414.htm] 